UCRL-JC-130835

PREPRINT

\title{
Impact of Detailed Radiation Transport on Volume Recombination
}

\author{
H. A. Scott, A. S. Wan, D. E. Post, M. E. Rensink, T. D. Rognlien
}

This paper was prepared for submittal to the 13th International Conference on Plasma Surface Interactions

San Diego, CA

May 18-22, 1998

May 19, 1998

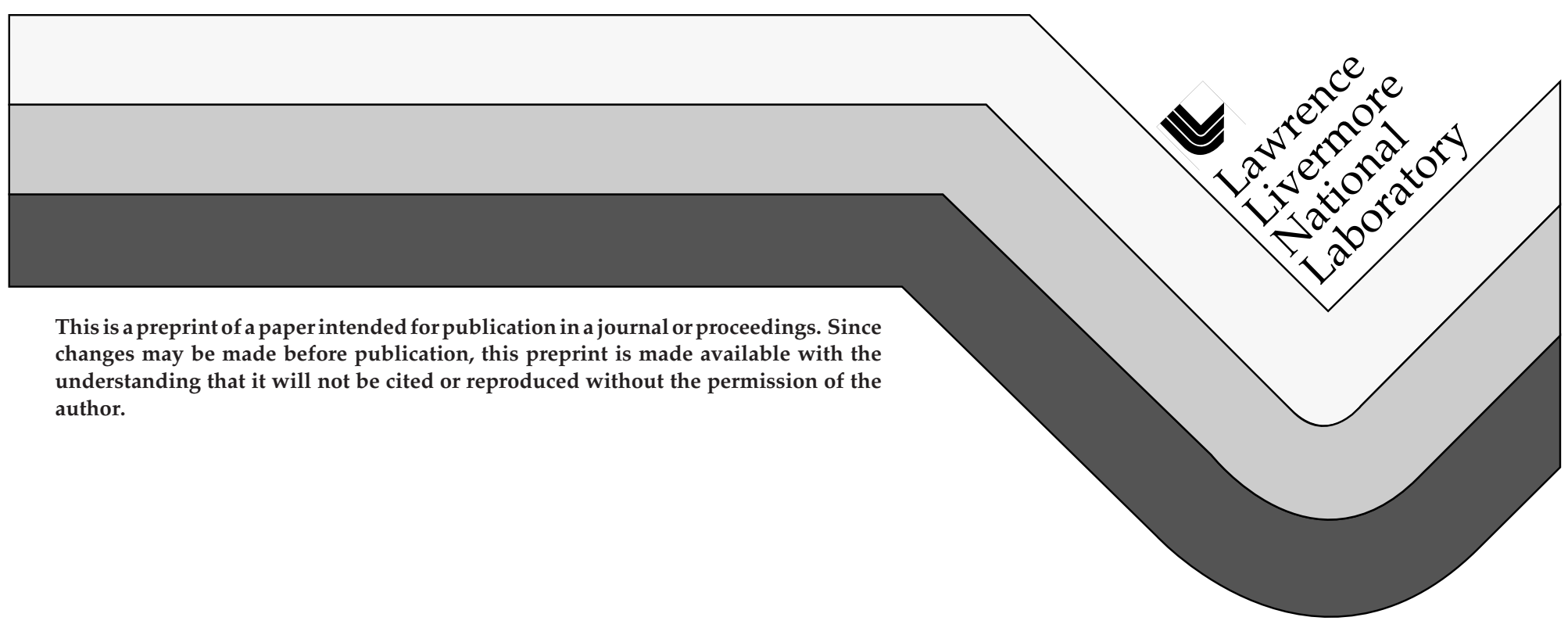




\section{DISCLAIMER}

This document was prepared as an account of work sponsored by an agency of the United States Government. Neither the United States Government nor the University of California nor any of their employees, makes any warranty, express or implied, or assumes any legal liability or responsibility for the accuracy, completeness, or usefulness of any information, apparatus, product, or process disclosed, or represents that its use would not infringe privately owned rights. Reference herein to any specific commercial product, process, or service by trade name, trademark, manufacturer, or otherwise, does not necessarily constitute or imply its endorsement, recommendation, or favoring by the United States Government or the University of California. The views and opinions of authors expressed herein do not necessarily state or reflect those of the United States Government or the University of California, and shall not be used for advertising or product endorsement purposes. 


\title{
Impact of detailed radiation transport on volume recombination
}

\author{
H. A. Scott, A. S. Wan, D. E. Post, M. E. Rensink, T. D. Rognlien
}

Lawrence Livermore National Laboratory, Livermore, CA 94551, USA

\begin{abstract}
:
Recently both the Alcator C-Mod and DIII-D tokamaks observed significant recombination of major ion species in the divertor region during detachment. For sufficiently low temperatures the mixture of neutral atoms and ions can be optically thick to line radiation. The optical depth of the recombined region to $\mathrm{Ly}_{\alpha}$ radiation can be very large and opacity effects and radiation trapping can dramatically change the heat flux to the divertor walls. This paper presents an analysis of the effect of line radiation on volume recombination using CRETIN, a multidimensional, non-local thermodynamic equilibrium simulation code that includes the atomic kinetics and radiative transport processes necessary to model this complex environment.
\end{abstract}

\section{Introduction}

In detached and/or highly radiating divertor conditions [1,2], achieving low temperatures [3,4] in the plasmas away from the material surface results in a significant amount of recombination of plasma ions and electrons. This insulates the divertor plate by reducing the incident ion flux to the material surface. The production of a strongly recombining region of major ion species in the divertor region away from the material surface is called "volume recombination" and has been 
observed recently on the Alcator C-Mod [5,6], ASDEX-Upgrade [7], JET [8], and DIII-D [9] tokamaks. However, to assess the impact of volume recombination and to be able to predict the degree of heat flux reduction to the divertor plate in a fusion reactor, we need a more detailed understanding of the phenomenology of this recombining region.

One of the major uncertainties in the ionization and recombination of plasmas away from the material surface is the effect of line radiation. The mean free paths of the Lyman lines are inversely proportional to the neutral population. For a neutral density of $\sim 10^{14} \mathrm{~cm}^{-3}$, the mean free path of a Ly $\alpha$ photon is $<0.2 \mathrm{~cm}$, which is significantly shorter than typical scrape-off-layer thickness. In a radiatively trapped case, line radiation can significantly alter both the excited state distribution and the ionization balance [10]. Radiative transitions produce an enhanced excited population, which can then be collisionally ionized. Even if the ionization balance is not changed significantly, opacity effects must be accounted for when calculating radiative energy fluxes or interpreting spectral information from optically thick lines.

Past edge modeling using collisional-radiative models used either optically thin or crude escape factor approximations. However, to assess the impact of line radiation in complex geometries where photons are absorbed and re-emitted in regions of varying optical depth and line profiles, simulations must also include line transport. The primary purpose of this paper is to present a sample calculation, using the non-local thermodynamics equilibrium (NLTE) code CRETIN, that illustrates how this can be used to assess the importance of line and continuum radiation in the recombining region of a divertor. We will address the collisional and radiative processes that are included in our atomic models which are important in the relevant regime in the divertor regions of present-day tokamaks. We then present a sample analysis of a two-dimensional (2-D) 
configuration representing the scrape-off layer (SOL) region just outside the magnetic separatrix with dimensions similar to the DIII-D tokamak. A UEDGE [11] simulation corresponding to low-power DIII-D operation, which easily yields a detached divertor plasma, provides the plasma profiles.

\section{Code Description and Relevant Atomic Physics}

CRETIN is a multi-dimensional NLTE simulation code [12] that includes the atomic kinetics and radiative transport processes necessary to model the complex environment of a divertor. Atomic processes include electron-ion and ion-ion collisions, photo-ionization and photo-excitation, and Auger processes. Rates for inverse processes are done via detailed balance. Radiative processes include treatments of bound-bound, bound-free, and free-free processes. CRETIN does not include hydrodynamics, but does include transport of neutral particles using a diffusion model [13]. Since the code does not transport ions, the diffusion is supplemented with the condition that the total mass density profile remains unchanged from the input profile. Transport of neutral particles can significantly affect the ionization balance near the divertor plate and is included in our analysis. The flux of the reentrant neutrals at the boundary is assumed to be proportional to the ion flux at the boundary.

\section{Case Study of a Radiative Divertor}

We present here a case study demonstrating the inclusion of radiation transport effects in simulating the DIII-D-like radiative divertor. Plasma density and temperature profiles calculated with the UEDGE [11] code were fed into CRETIN, which then calculated distributions of ions, 
atoms, and radiation. Neutral hydrogen was modeled with ten atomic states, characterized by principle quantum number, which were connected to every other state plus the hydrogen ion via collisional and radiative transitions. No molecular transitions were considered.

The geometry of a typical DIII-D-like tokamak with the SOL and divertor region is depicted in Figure 1(a). The SOL and divertor regions are idealized as a rectangular domain for this initial investigation. The UEDGE plasma simulation is performed over a 100-cm poloidal length corresponding to the outer half of the SOL/divertor regions with a distance of $25 \mathrm{~cm}$ between the magnetic X-point and the divertor plate. CRETIN then uses the plasma solution in the X-pointto-plate subdomain as depicted in Fig. 1(b) to do the radiation transport calculation. A more realistic geometry will be used in future studies.

The configuration chosen for study corresponds to a detached plasma with a moderately cold dense plasma (where radiation trapping should be important) extending roughly halfway to the X-point. The plasma electron temperatures and number densities, as calculated by UEDGE, are shown in Figures 2 and 3. The boundary conditions for the CRETIN simulation were: 1) radiation could freely escape from all boundaries, 2) zero flux of neutral particles through the walls and back towards the main plasma, and 3) a recycling condition at the divertor plate, where ions hitting the plate are reintroduced as neutrals.

For these densities and temperatures, the cold plasma is very optically thick to Ly $\mathrm{y}_{\alpha}$ radiation. The optical depth from the X-point to the plate is of order $10^{3}$. The optical depth between the walls through the cold plasma is also of order $10^{3}$. Most of the $\mathrm{Ly}_{\alpha}$ emission occurs on the edge of the cold dense region. Figure 4 shows the distribution of the $n=2$ excited state population, 
which is proportional to the emission coefficient. The temperature quickly becomes high enough towards the main plasma so that line photons can freely escape, but the flux of line photons towards the wall is severely attenuated.

The radiation trapping and optical depth effects are quite important for understanding the performance of the divertor. Figure 5 shows the radiative energy flux along the divertor plate which is primarily ( $90 \%)$ due to continuum radiation. The solid line is from an optically-thin calculation that neglected attenuation of the $\mathrm{Ly}_{\alpha}$ radiation, while the dashed line is from a calculation that self-consistently treated the transport of $\mathrm{Ly}_{\alpha}$ radiation through the cold plasma, including both absorption and re-emission. The optically thin calculation overestimates the radiative flux to the divertor plate due to line radiation by two orders of magnitude, and overestimates the total radiative flux by two orders of magnitude. When attenuation is included, $90 \%$ of the radiation reaching the plate is from continuum emission.

The dot-dashed line in Figure 5 is from a calculation that accounted for attenuation of the line through the cold plasma, but did not include re-emission. The difference is due to radiation trapping effects in the cold plasma. The trapped radiation results in $\sim 10 \%$ higher in electron densities which produces $\sim 20 \%$ more recombination radiation.

Figure 6 shows the radiative energy flux along the inner wall $(y=-1 \mathrm{~cm}$ in the simplified geometry). The dotted line gives the total flux from an optically-thin calculation, while the solid line gives the total flux from a self-consistent calculation. Again, the optically thin calculation overestimates the total flux by two orders of magnitude. The dashed and dot-dashed curves 
represent the contributions of continuum and $\mathrm{Ly}_{\alpha}$ radiation, respectively. Radiation trapping does not significantly affect these results since most of the flux is still due to line radiation.

The calculations performed by CRETIN are self-consistent with respect to the Ly $\mathrm{y}_{\alpha}$ radiation and the ionization balance and excited population distribution. However, the temperatures and total plasma densities were calculated by UEDGE under the assumption that the plasma was optically thin and radiation would escape. Since this is far from consistent with the results of the radiation transfer calculations, the energy balance in the divertor region may be quite different than that obtained by UEDGE. Future work will focus on providing a method to address this problem.

The geometry and non-uniform distribution of populations also affects the interpretation of spectral diagnostics, since a given line of sight will pass through regions of differing emission and opacity. Figure 7 shows a simulated spectrum that would be observed by a detector oriented as pictured in Figure 1(b). The crosses indicate the values that would be predicted by an optically thin calculation. Attenuation significantly affects the peaks corresponding to the first four members of the Lyman series (above $10 \mathrm{eV}$ ). The amount of attenuation will depend on the configuration of the plasma with respect to the detector. The line wings are optically thin and unaffected by attenuation, but will still depend on the particular region of plasma sampled by the detector.

\section{Summary}

Recombining divertor plasmas can quickly become optically thick to line radiation. We have done some preliminary NLTE radiative transfer calculations for a simplified DIII-D 
configuration simulated with UEDGE to assess the consequences of this fact. The very large attenuation of the line radiation can dramatically alter the energy balance in the divertor region, with a much smaller impact from radiation trapping. A full evaluation of this effect will require a self-consistent simulation of the divertor plasma that includes radiative transfer.

\section{Acknowledgments}

This work is performed under the auspices of the U. S. DOE by LLNL under the contract number W-7405-ENG-48.

\section{References}

[1] G.F. Matthews J. Nuclear Material, 220-222 (1995) 104-116.

[2] A. Loarte, J. Nuclear Material, 241-243 (1997) 118-134.

[3] B. LaBombard et al., Phys. Plasmas, 2 (1995) 2242.

[4] S.L. Allen et al., J. Nuclear Material, 241-243 (1997) 595-601.

[5] J.L. Terry et al., to be published in Phys. Plasmas.

[6] D. Lumma, J.L. Terry, and B. Lipschultz, Phys. Plasmas, 4 (7), 1997-2555.

[7] B. Napiontek et al., Proc. of the 24th European Phys. Soc. Conf. on Contr. Fus. and Plasma Phys., 21A-part III European Physical Society, Petit-Lancy (1997) 1225.

[8] G.M. McCracken et al., submitted to Nuclear Fusion.

[9] R. Isler et al, Phys. Plasmas, 4 (1997) 2989.

[10] A.S. Wan, H.E. Dalhed, H.A. Scott, D.E. Post, T.D. Rognlien,J. Nuclear Material, 220222 (1995) 1102-1106. 
[11] T.D. Rognlien, J.L. Milovich, M.E. Rensink, G.D. Porter, J. Nuclear Material, 196-198 (1992) 347.

[12] H.A. Scott and R.W. Mayle, Applied Phys. B 58 (1994) 35.

[13] T.D. Rognlien et al., Contr. Plasma Physics 34 (1994) 362 


\section{Figure Caption}

Figure 1. Rectangular geometry used in this study (b) is an idealized simplification of a more realistic $2 \mathrm{D}$ divertor shown in (a). Also shown in (b) are the detector position and orientation used to calculate the spectrum shown in Figure 7.

Figure 2. Contours of constant electron temperature (in $\mathrm{eV}$ ) in the divertor region from a UEDGE calculation. This temperature distribution, and a similar distribution for ion temperature, was held constant for the radiative transfer calculation.

Figure 3. Contours of constant hydrogen number density (ions + neutrals, in units of $10^{14} \mathrm{~cm}^{-3}$ ) in the divertor region from a UEDGE calculation. This distribution was held constant for the radiative transfer calculation, although the ionization balance and excited state distributions were allowed to vary.

Figure 4. Contours of constant number density of the $n=2$ excited state of neutral hydrogen (in units of $10^{10} \mathrm{~cm}^{-3}$ ) from the CRETIN calculation. This quantity is proportional to the $\mathrm{Ly}_{\alpha}$ emission coefficient.

Figure 5. Radiative energy flux incident upon the divertor plate as a function of position along the plate. The solid curve assumes that the plasma is optically thin to $\mathrm{Ly}_{\alpha}$ radiation. The dot-dashed curve accounts for absorption of the radiation by intervening plasma. The dashed curve includes both absorption and re-emission of the $\mathrm{Ly}_{\alpha}$ radiation. 
Figure 6. Radiative energy flux incident upon the bottom wall $(\mathrm{y}=-1 \mathrm{~cm}$.) as a function of position along the wall. The dotted curve assumes that the plasma is optically thin to $\mathrm{Ly}_{\alpha}$ radiation. The solid curve includes both absorption and re-emission of the Ly $\alpha$ radiation. The dot-dashed and dashed curves show the portion of the flux carried by $\mathrm{Ly}_{\alpha}$ and continuum radiation, respectively.

Figure 7. Simulated spectrum which would be observed by a detector with an acceptance of 0.1 steradian placed at $(\mathrm{x}=95 \mathrm{~cm}, \mathrm{y}=4 \mathrm{~cm})$ and oriented 15 degrees below the $\mathrm{x}$-axis (see Figure $1 \mathrm{~b})$. The detector is assumed to have a flat response curve and negligible broadening. The X's mark the peak flux densities that would be predicted for the Lyman line and Balmer line series in the absence of absorption. 
(a)

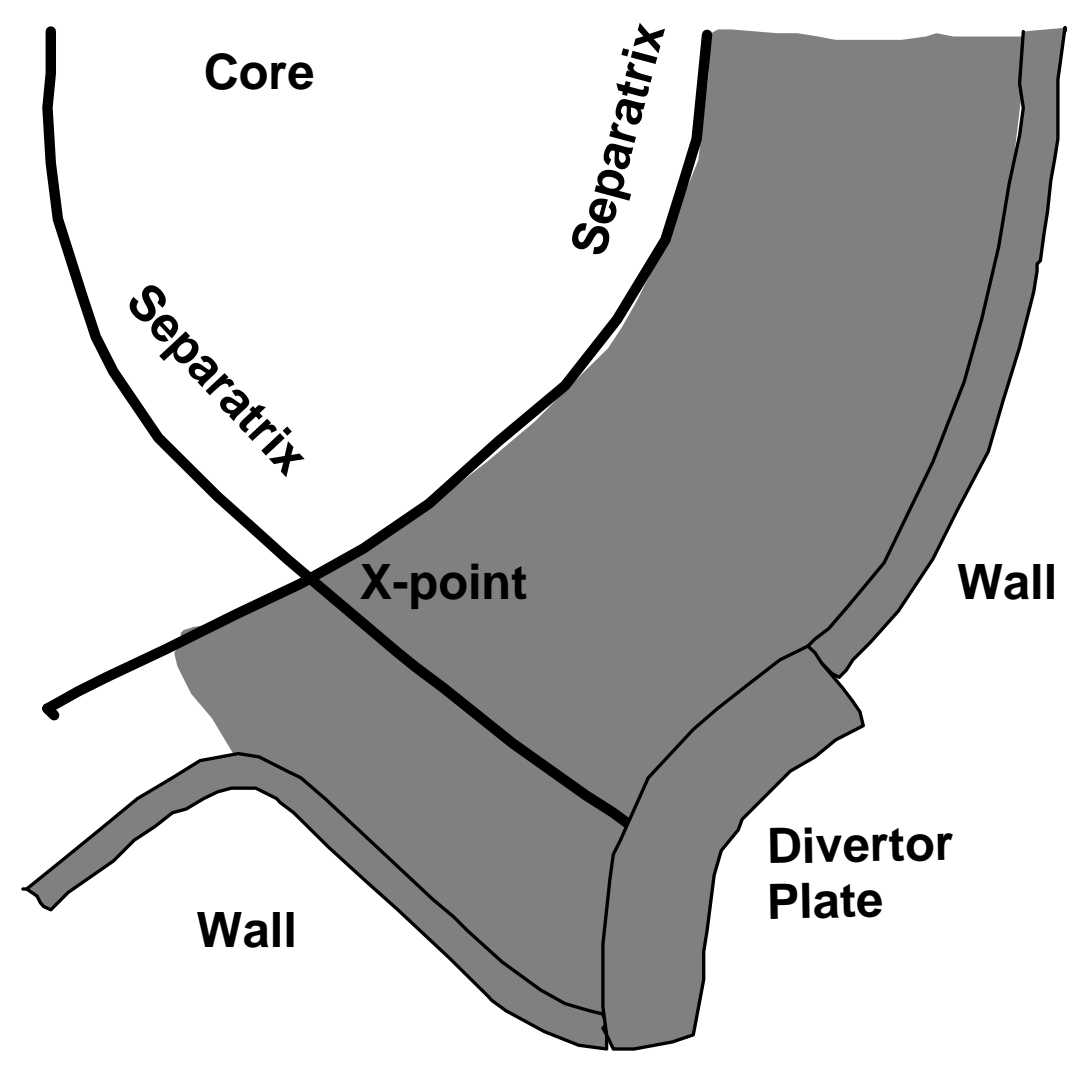

(b)
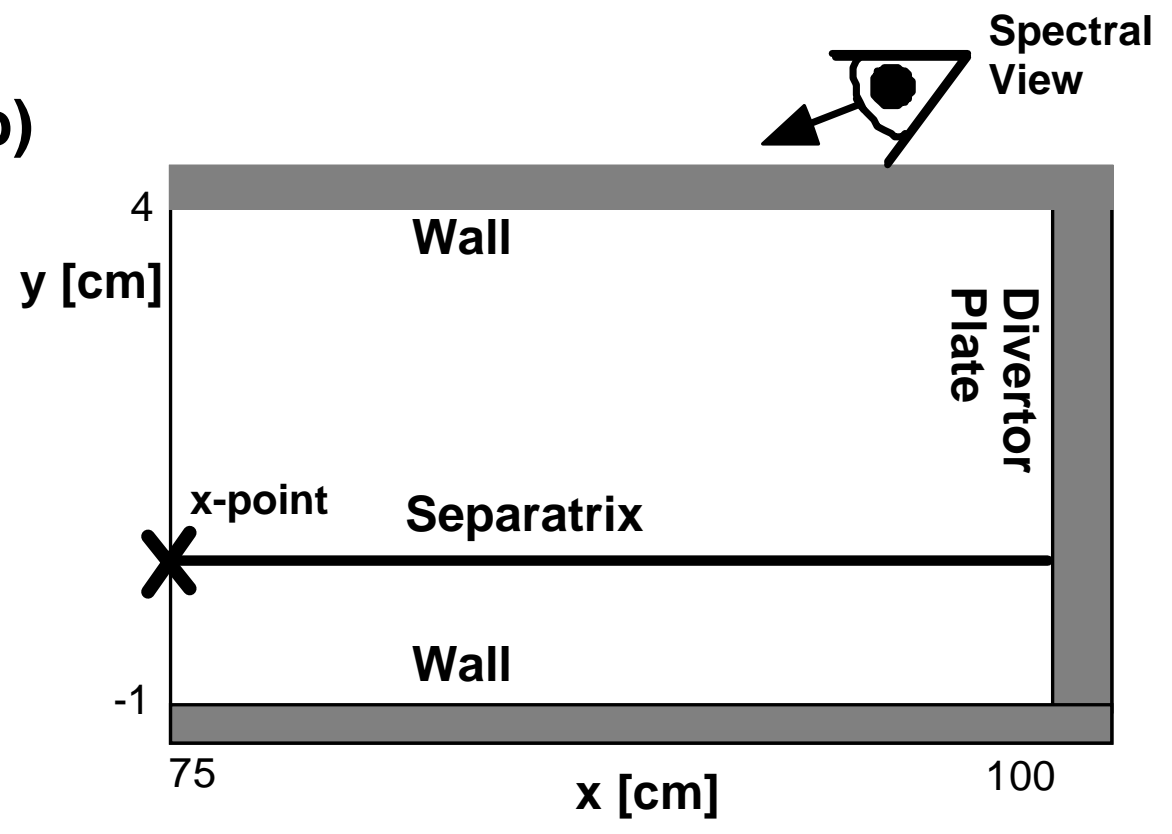

Figure 1. 


\section{Electron temperature (eV) from UEDGE calculation}

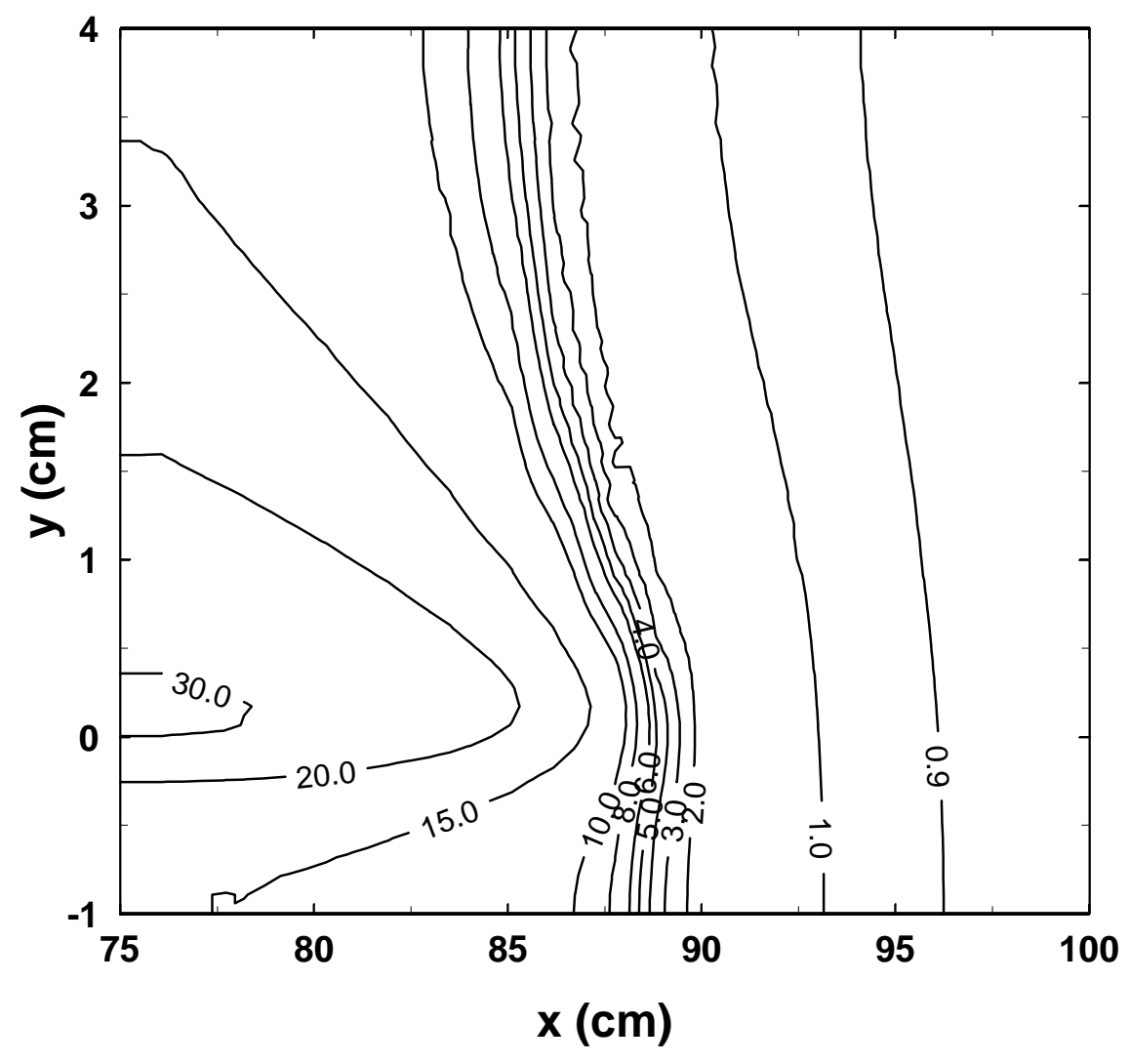

Figure 2. 


\section{Number density $\left(10^{14} \mathrm{~cm}^{-3}\right)$ from UEDGE calculation}

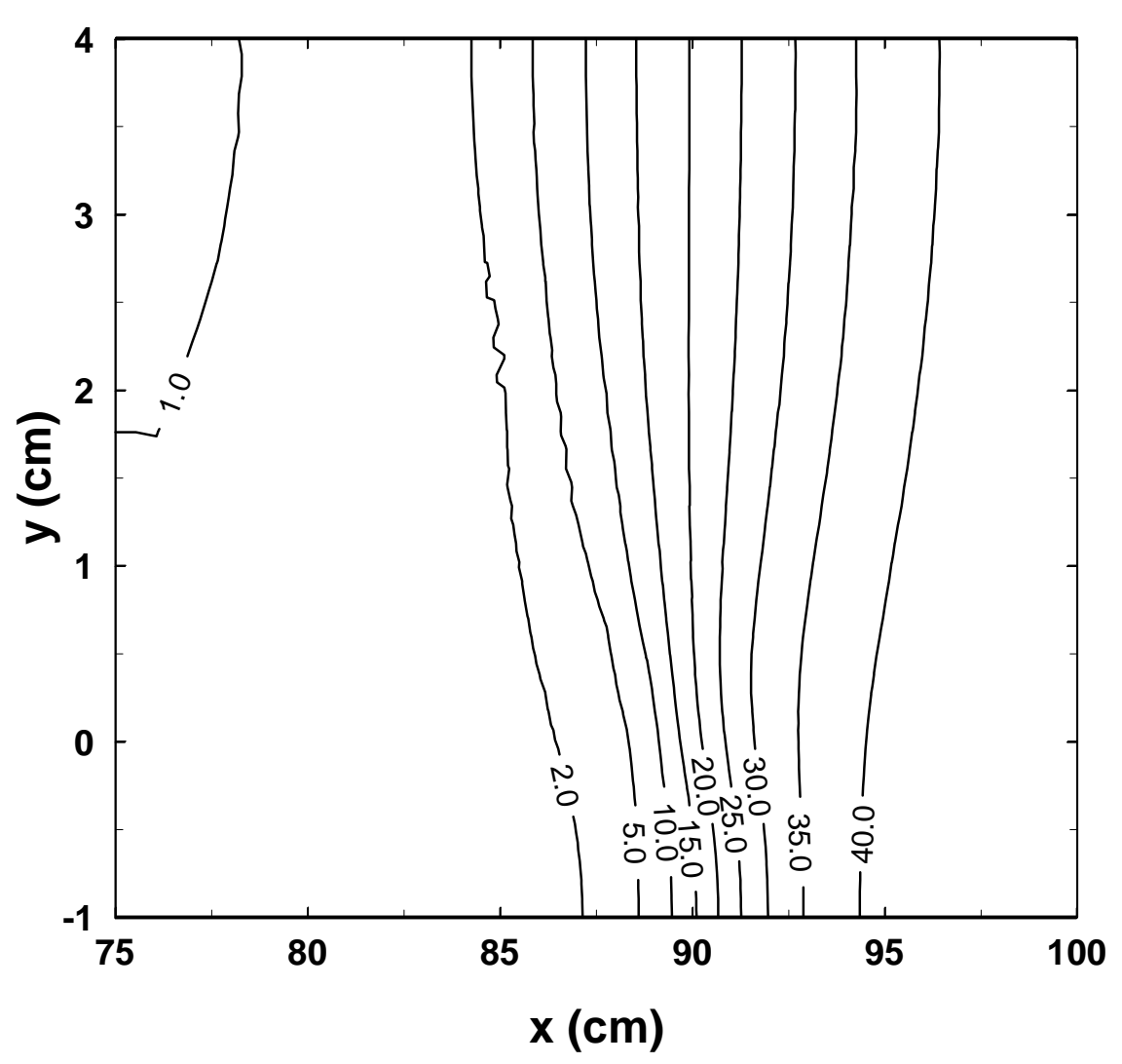

Figure 3. 

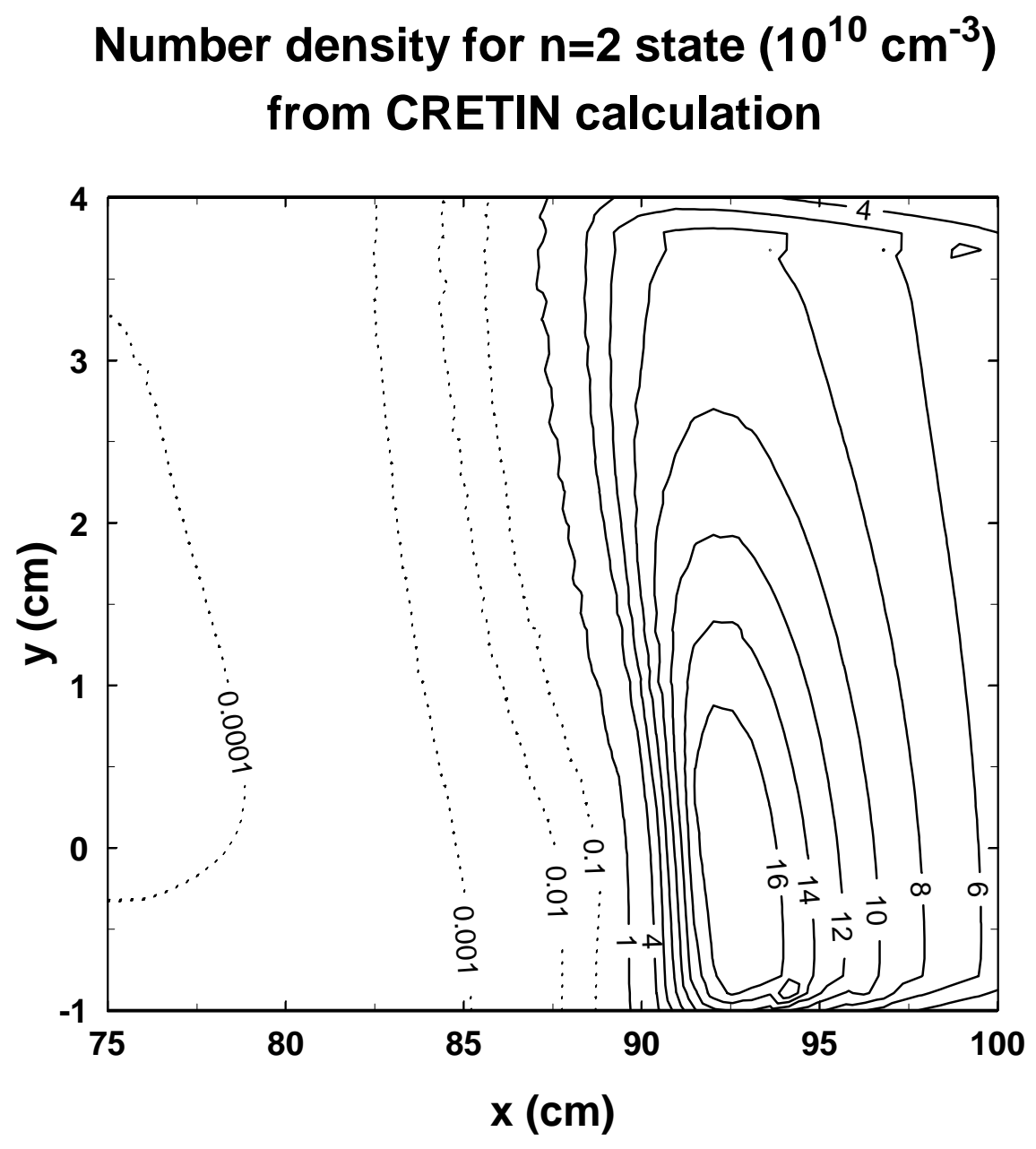

Figure 4. 


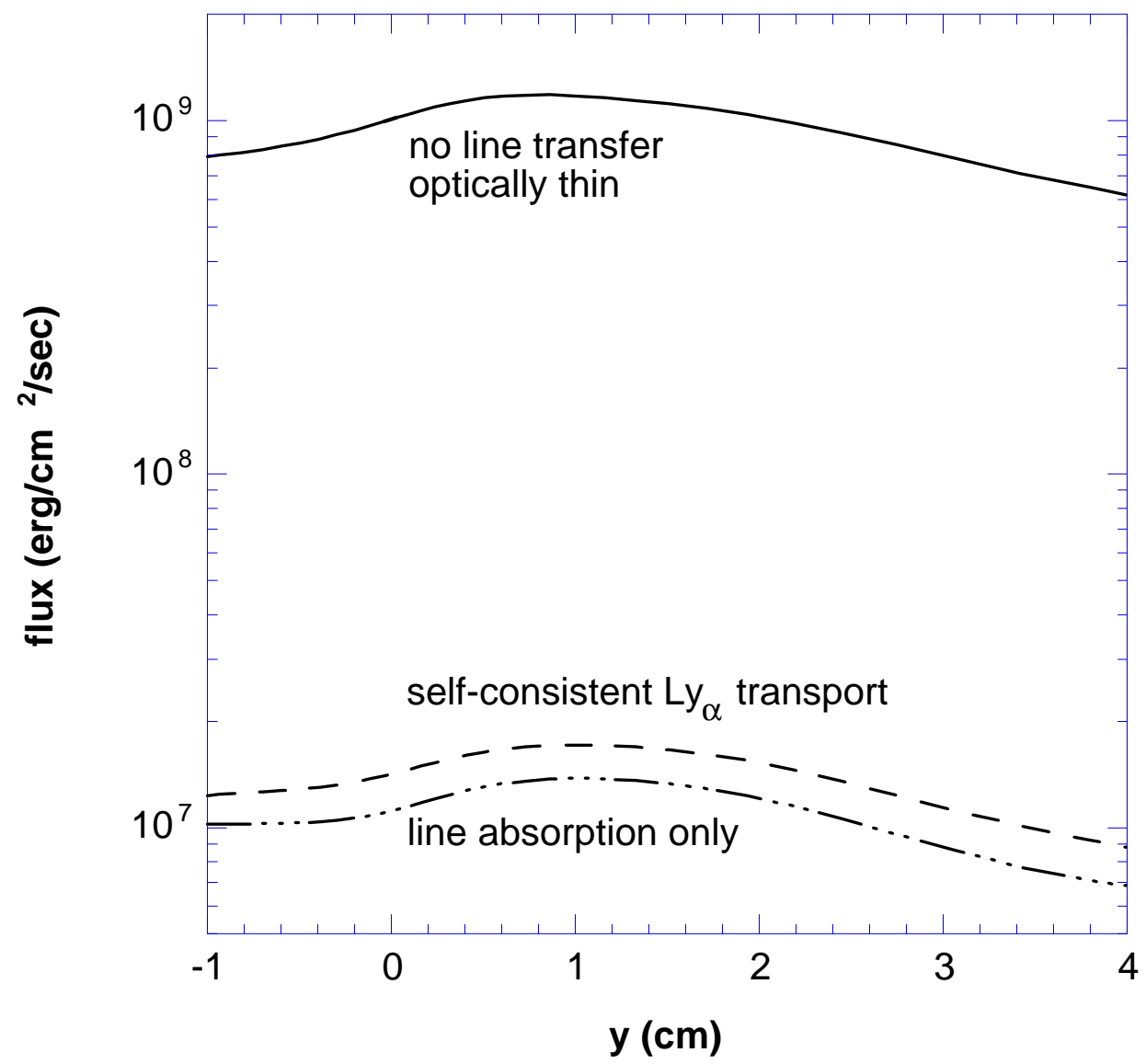

Figure 5. 


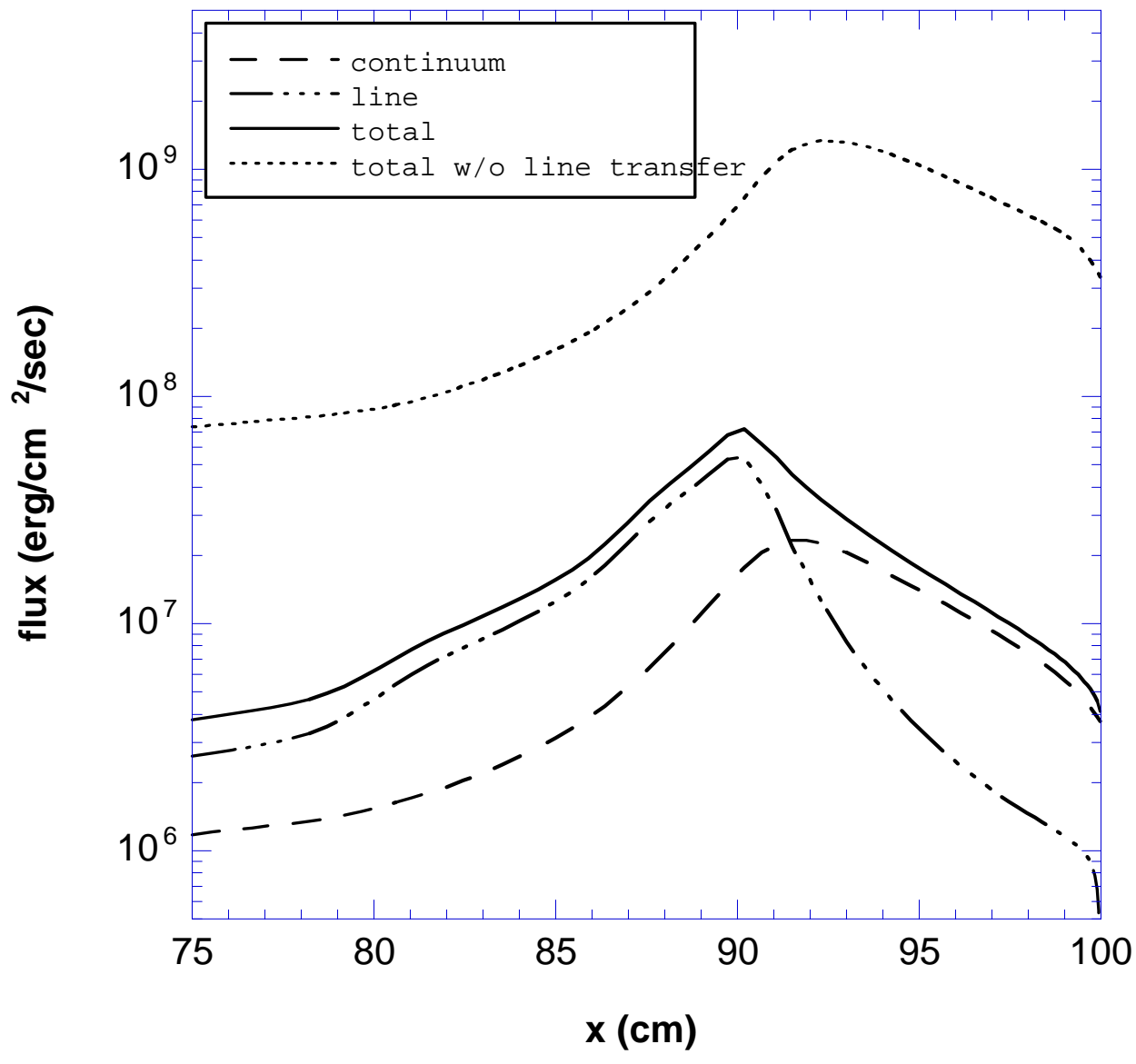

Figure 6. 


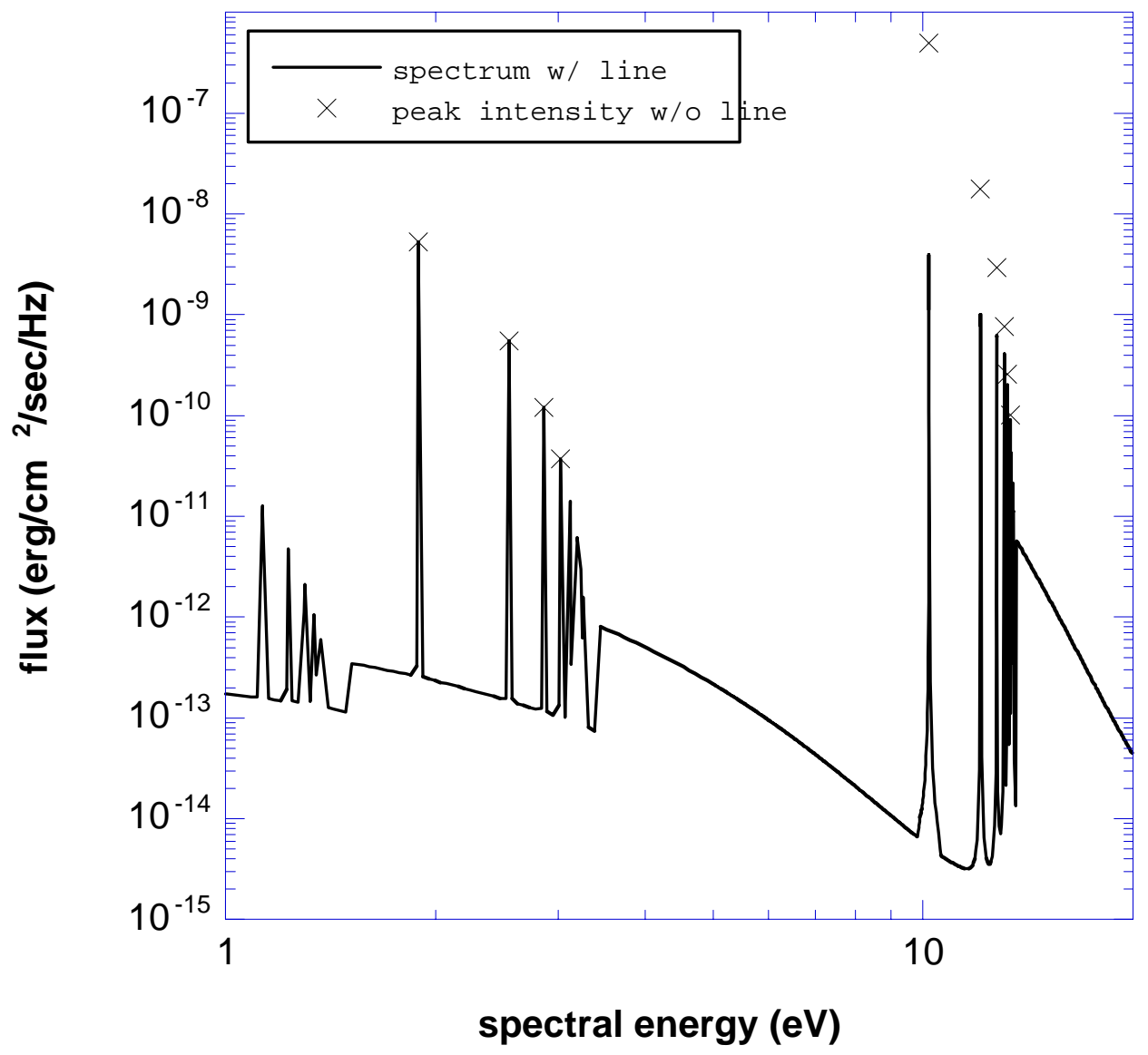

Figure 7. 


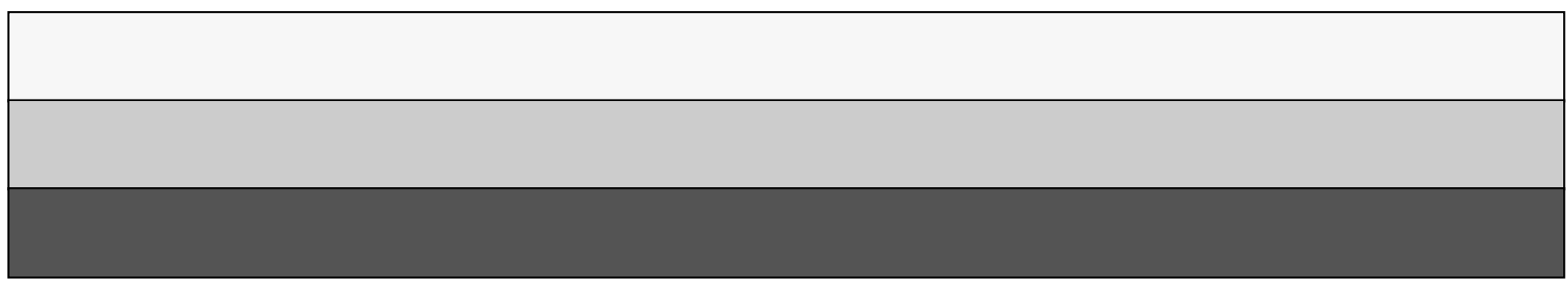

